\title{
A New Role for Coprocessors
}

\section{Dear Reader,}

those who look back with fondness on the good old days when x87 coprocessors made their market debut may know the thrill of tuning a computer and experiencing unprecedented computational speeds. Somewhat later, the next level to be reached was a Graphics Processing Unit (GPU) often repleted with a do-it-yourself aquarium-pump-driven cooling. In our current times of truly high-performance pocket calculators, also known as smartphones, such retrospection may come across like a quaint attempt to make the ancient legends of the 1990s more heroic.

And yet, the notion of auxiliary, dedicated processors has returned to the spotlight, since it makes perfect sense as developers pursue alluring applications such as highly automated driving. The sequestering of specific functions to dedicated processors or cores can enable tailor-made and highly energyefficient computing systems. Indeed, the very principle is essential when it comes to further modularization and a central computer architecture in vehicles. According to Texas Instruments, a sensible combination of hardware accelerators and programmable cores is as important for the quality of a computer solution as the generated performance data are for control tasks.

In addition to computer architecture, the development and validation of software for automated functions is more than an important side issue. The typical sequence whereby software development precedes hardware development makes for twice the work. A software developed using any given computer hardware located in the trunk of a test vehicle will still need to be set up on the vehicle computer to be specified, and will need to be validated again. Operationalizing the notion that a development system does not equal a runtime system prolongs the process and leaves developers facing the risk of obsolescence.

The only conclusion is that both need to go hand in hand. OEMs and development service providers also need to work closely together with the hardware manufacturers to ensure the development of appropriately sophisticated computer hardware. This is the only way to ensure the production of state-of-the-art computer architectures for start-of-production vehicles. The trick is to stay close to the consumer product so as to exploit the advantages of mass production while also creating vehicle-specific architectures. "Automotive coprocessors" may represent an technology-neutral, modular path to this goal.

We hope you enjoy reading this latest issue!

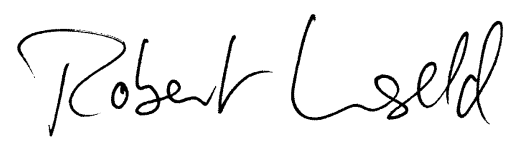

Robert Unseld

Responsible Editor

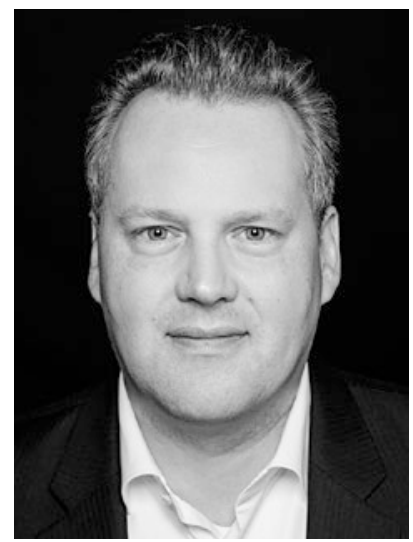

\section{Tutoring for a car?}

Cognitive skills for best-in-class performance.

Autonomous driving is coming up fast. But a technology as sophisticated as this demands so much of vehicles. They have to learn to recognize situations, make decisions on their own, and act at precisely the right moment. Take advantage of our cognitive approach and 25 years' experience in control engineering. Let's work together to develop best-in-class solutions that excel in every dilemma test.

ITK Engineering GmbH - Putting the smarts into smart machines.

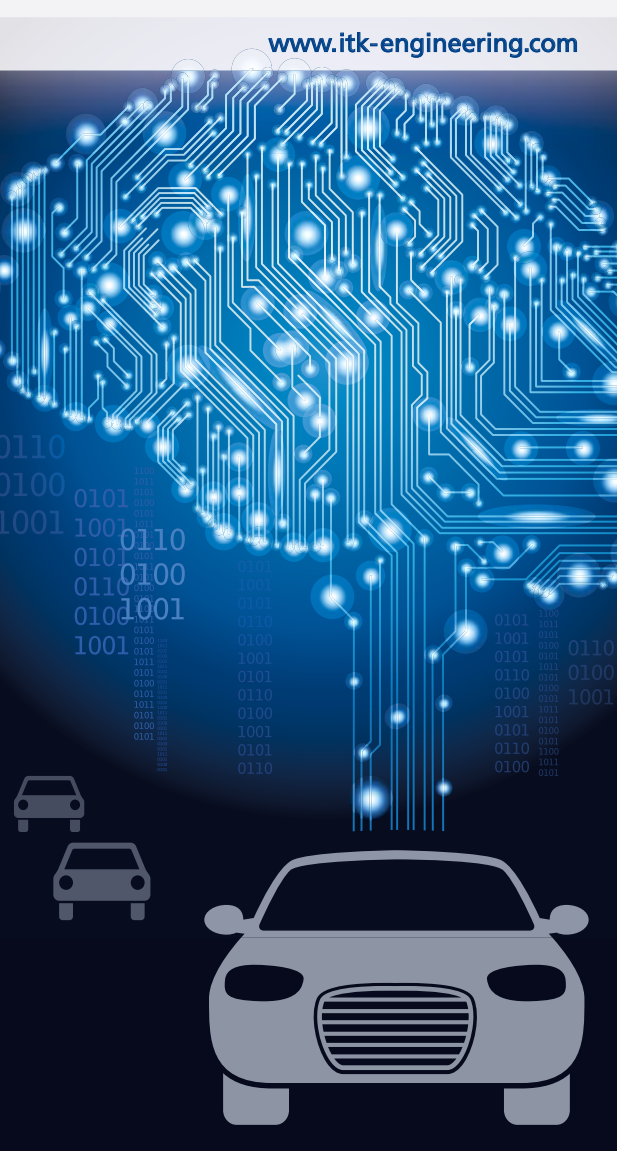

